$16^{\text {th }}$ International Congress of Metrology, 10002 (2013)

DOI: $10.1051 /$ metrology/201310002

(c) Owned by the authors, published by EDP Sciences, 2013

\title{
European project: Metrology for Chemical Pollutants in Air
}

\author{
Annarita Baldan ${ }^{1, \mathrm{a}}$ \\ ${ }^{1}$ VSL, Thijsseweg 11, 2629JA Delft, The Netherlands
}

\begin{abstract}
The measurement of gaseous pollutants in air is a sensitive and priority issue that has a large impact on human health and environment. The European Directive on "Ambient air quality and cleaner air for Europe" $(2008 / 50 / E C)$ sets limit values and data quality objectives for the measurement in EU member states of air pollutants in ambient air. Although no harmonized legislation is currently present for the monitoring of indoor air, the concern on the exposure to indoor chemical pollutants is clearly increasing. The Joint Research Project MACPoll (Metrology for Chemical Pollutants in Air), running under the European Metrology Research Program, addresses the need to improve the metrological traceability and comparability of measurements using current air monitoring techniques and the need to set up a metrological bases for the new sensor technologies used in air quality applications.
\end{abstract}

\section{Introduction}

Chemical air pollutants are gaseous or vapour compounds in air that are potentially harmful with respect to human health and to the environment. The monitoring of air pollution, air quality monitoring, focuses on the measurement of these compounds in ambient and indoor air.

Ambient air is outdoor air of the troposphere. The concentration levels of the primary gas pollutants (sulphur dioxides, nitrogen oxides, carbon monoxides, benzene and ozone) are regulated by the European Directive 2008/50/EC on air quality [1]. These gases are the result of human activities (such as exhaust vehicles in cities) and industrial emissions or natural processes (such as volcanic eruptions).

Indoor air is air in a confined space, such as in buildings. High priority gas indoor pollutants are various volatile organic compounds, formaldehyde, carbon monoxide, nitrogen dioxide and ozone. The regulation and assessment of indoor chemical pollution is very difficult, due to the infiltration of polluted outdoor air and to emissions from countless sources, such as building materials, furniture, human activities, etc.

In support to the preparation and enforcement of air quality regulation, standardization and metrological activities are deemed of fundamental importance. They give the basis, by complementing each other, to a proper measurement infrastructure.

The European Joint Research Project: Metrology for chemical pollutants in air (MACPoll) [2] aims at providing key parts of the metrological backbone that

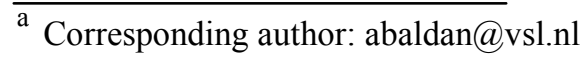

will enable accurate and robust data on air pollution levels for short and long (trend) term assessment.

In particular, the JRP focuses on improving the metrological traceability and comparability of measurements using current air monitoring techniques and on setting-up a metrological basis for the new sensor technologies used in air quality applications.

\section{Outline of JRP MACPoll}

MACPoll is a three year project and it was launched on the 1st of June 2011. This JRP runs under the European Metrology Research Program and it receives European funding.

In total 12 EU Metrology Institutes, 3 Researchers Excellence Grants and 2 Research Mobility Grants are working side by side in research activities related to ambient and indoor air.

\subsection{Calibration gases for ambient air pollutants}

One of the main objectives of this project is to comply with the challenging requirements of the calibration gases, both for span and for zero gas, set in documentary EN standards related to the air quality directive (2008/50/EC) reference methods.

\subsubsection{Calibration gases for nitrogen oxides and sulphur dioxides at Limit Values}

The lack of stable Certified Reference Materials (CRM) in cylinders for reactive gases at the limit values (parts per billion levels) mandated by the air quality directive 
limits the possibility of offering traceable gas mixtures for the calibration of analysers in air quality measurements. Alternative traceable methods for the preparation of gas mixtures, such as dynamic and static dilution, are considered a proper alternative.

The current investigation of these preparation methods for nitrogen oxides $\left(\mathrm{NO}_{\mathrm{x}}\right)$ and sulphur dioxide $\left(\mathrm{SO}_{2}\right)$ aims at providing the degree of comparability and of uncertainty of these techniques and their suitability for implementation at monitoring networks.

\subsubsection{Assessing impurities in zero gas}

Nitrogen and pure air, used for zeroing gas analysers and for dilution purposes, should be free of contaminants that may interfere with the measurements. Special attention is dedicated to the relevant zero gas impurities that affect the measurement of nitrogen oxides and sulphur dioxide. These impurities have to be minimized to (sub)-ppb level and their presence quantified. The measurement approach chosen is the single or simultaneous assessment by means of optical systems, such as Cavity Ring Down Spectroscopy (CRDS) or Tunable Diode Laser Adsorption Spectroscopy (TDLAS). Once this measurement approach is fully traceable, a protocol for the certification of the impurity composition of zero gas will be developed.

\subsection{Reference systems for indoor air pollutants}

The needs identified in indoor air pollution are related to the development of references systems for (semi) volatile organic compounds.

The JRP is currently improving reference methods and developing reference materials with special attention to the semi-volatile organic compounds ((S)VOC), harmful compounds originating from emission of building materials, for which not much information on sampling and measurement systems is available yet.

In addition, the production of a constant emitting reference material for the quality control of emission test chamber measurements is under investigation.

\subsection{Micro sensors for air monitoring applications}

Air Quality assessment in the future will rely on more spatial and exposure based monitoring of air pollutants (indicative measurements). The need for mobile applications and better spatial coverage can only be satisfied in reducing size and costs of monitoring devices using for example micro-sensors.

Present technology has allowed the introduction on the market of various micro-sensors (solid state, electrochemical, fibre optical, etc.) for air monitoring, however, the validation carried out so far lack of a structured and metrological approach.

One of the goals is then to carry out the validation of commercial sensor systems and in particular the metrological aspects (traceability and uncertainty of the results).
In parallel, a key deliverable of this JRP consists of developing a new highly sensitive and selective $\mathrm{NO}_{2}$ sensor using the promising two- dimensional material graphene.

The following institutes: BAM (D), METAS $(\mathrm{CH})$, FMI (FI), INRIM (I), JRC (Institute for Environment and Sustainability, EC), LNE (F), MIKES (FI), NPL (UK), PTB (D), SMU (SK) and UBA (D) are forming the consortium under the coordination of VSL (NL). In addition to the consortium, three researchers, respectively from the Spanish Research Council (CSIC), from the University of Helsinki and from the University of Aalto, are contributing to the research programme with a high level expertise. Within the project, two metrologists from respectively the Turkish and the Serbian NMIs are trained by the consortium to perform research in specific project activities.

All project partners are active in the field of air quality and their key-scientists represent the top European expertise in air quality, gas analysis, spectroscopy, preparation of gaseous Certified Reference Materials and testing and validation.

\section{Calibration gases for reactive air pollutants at EU Limit Values}

This part of the project focuses on traceable calibration gases for nitrogen dioxide $\left(\mathrm{NO}_{2}\right)$ and sulphur dioxide $\left(\mathrm{SO}_{2}\right)$ at concentration levels corresponding to the Limit Values (LVs) given in the Air Quality Directive $(2008 / 50 / \mathrm{EC})$ for the measurement of ambient air pollutants. Tables 1 and 2 give an overview of the EU LVs. Nitrogen oxide (NO) is normally measured in combination with nitrogen dioxide for the measurement of $\mathrm{NO}_{\mathrm{x}}$. Although $\mathrm{NO}$ is a reactive gas, reference standards at air quality concentration levels have proven to be quite stable. Therefore NO is used as test gas to validate the activities of this research.

Details of this work can be found in the web conference publication 'Calibration gases for existing air quality directive pollutants at Limit Values (LVs)' under the $16^{\text {th }}$ International Congress of Metrology [3].

Table 1. EU Limit Values for $\mathrm{SO}_{2}$.

\begin{tabular}{|c|c|}
\hline $\mathbf{S O}_{2}$ & $\begin{array}{c}\text { Limit Values } \\
(\mathbf{L V}), \\
\mathbf{n m o l} / \mathbf{m o l}\end{array}$ \\
\hline One hour & $132^{1}$ \\
\hline One day & $47^{1}$ \\
\hline $\begin{array}{c}\text { Calendar } \\
\text { year }\end{array}$ & $8^{2}$ \\
\hline
\end{tabular}


Table 2. EU Limit Values for $\mathrm{NO}_{2}$.

\begin{tabular}{|c|c|}
\hline $\mathbf{N O}_{\mathbf{2}}$ & $\begin{array}{c}\text { Limit Values } \\
(\mathbf{L V}), \\
\mathbf{n m o l} / \mathbf{m o l}\end{array}$ \\
\hline One hour & $105^{1}$ \\
\hline $\begin{array}{c}\text { Calendar } \\
\text { year }\end{array}$ & $21^{1}$ \\
\hline
\end{tabular}

${ }^{I}$ Air quality directive (2008/50/EC) Annex XI and art. 13

${ }^{2}$ Air quality directive (2008/50/EC) Annex XIII and art. 14

\subsection{Dynamic generation methods}

The generation methods selected for preparation of calibration gases of $\mathrm{NO}, \mathrm{NO}_{2}$ and $\mathrm{SO}_{2}$ at LVs are described in ISO 6144 [4] and ISO 6145 [5] documentary standards.

The so called 'dynamic dilution' technique makes use of mass flow controllers or molbloc/molbox systems to dilute high concentrated gas standards in high pressure cylinders to the required concentrations. Dynamic dilution appears to be a good method for the three reactive gases under investigation. The 'permeation' technique has been successfully applied in the past for $\mathrm{NO}_{2}$ and $\mathrm{SO}_{2}$. This study aims at improving the uncertainty of this technique when applied to reach the low part-per-billions ( $\mathrm{ppb}$ ) concentrations close to the LV. The third, the 'static dilution' technique, is based on the injection of a known volume of gas into a vessel with well characterised volume. This method is used to obtain $\mathrm{NO}$ and $\mathrm{SO}_{2}$ gas standards.

\subsection{Comparing calibration gases}

A comparison exercise is currently carried out within MACPoll to validate the above described preparation techniques. The target is set so that the expected degree of comparability for $\mathrm{NO}$ and $\mathrm{SO}_{2}$ calibration gases, at concentrations close to the LVs, should be better than $2 \%$ and for $\mathrm{NO}_{2}$ better than $3 \%$.

A thorough study has been performed on the selection, built up and characterisation of the travelling standards needed for the comparison. Normally gas mixtures in high pressure cylinders are the preferred choice for comparisons in gas analysis. However, the lack of gas standards at the concentration required has deemed to look at alternatives. In this comparison, two types of travelling standards have been developed. For $\mathrm{NO}_{2}$, a portable generation system based on permeation and flow dilutor, has been build up by the Swiss Metrology Institute METAS. For $\mathrm{NO}$ and $\mathrm{SO}_{2}$, a portable dilution system based on molbloc/molbox was constructed by LNE. These travelling standards fulfil the requirements of robustness, stability and low uncertainty.

\section{Zero gas standards for the measurement of reactive pollutants}

The zero gas under investigation is clean nitrogen or clean air which is used in air quality network both for zeroing the analyser that measure the pollutants and for generating the span gas (gas standard) that is used as calibration point.

More specifically, the zero gas of interest in this JRP is the one suitable for the measurement of reactive gases. Critical impurities in zero gas are therefore trace levels of the reactive gases under measurement and the compounds that may interfere in the measurement. The list of impurities and their specifications (maximum concentration levels) are described in the standards EN 14211 [6] and EN 14212 [7] prepared by CEN TC264/WG12 "Reference method for the determination of $\mathrm{SO}_{2} / \mathrm{NO}_{2} / \mathrm{O}_{3} / \mathrm{CO}$ in ambient air". Tables 3 and 4 give an overview of the impurities in zero gas for the measurement of $\mathrm{SO}_{2}$ and $\mathrm{NO}_{\mathrm{x}}$ respectively.

Table 3. Impurities in zero gas used for $\mathrm{SO}_{2}$ measurements (EN 14212).

\begin{tabular}{|c|c|}
\hline Pollutant & $\begin{array}{c}\text { Concentration } \\
\boldsymbol{\mu m o l} / \mathbf{m o l}\end{array}$ \\
\hline $\mathrm{H}_{2} \mathrm{~S}$ & $\leq 0.1$ \\
\hline $\mathrm{NH}_{3}$ & $\leq 0.01$ \\
\hline $\mathrm{NO}$ & $\leq 0.001$ \\
\hline $\mathrm{NO}_{2}$ & $\leq 0.001$ \\
\hline $\mathrm{Water}$ & $\leq 150$ \\
\hline $\mathrm{SO}_{2}$ & $\leq 0.001$ \\
\hline $\mathrm{m}-\mathrm{Xylene}$ & $\leq 0.001$ \\
\hline
\end{tabular}

Table 4. Impurities in zero gas used for $\mathrm{NO}_{\mathrm{x}}$ measurements (EN 14211).

\begin{tabular}{|c|c|}
\hline Pollutant & $\begin{array}{c}\text { Concentration } \\
\boldsymbol{\mu} \text { mol/mol }\end{array}$ \\
\hline $\mathrm{CO}_{2}$ & $\leq 400$ \\
\hline $\mathrm{O}_{3}$ & $\leq 0.002$ \\
\hline $\mathrm{NH}_{3}$ & $\leq 0.01$ \\
\hline $\mathrm{NO}$ & $\leq 0.001$ \\
\hline $\mathrm{NO}$ & $\leq 0.001$ \\
\hline $\mathrm{Water}_{2}$ & $\leq 150$ \\
\hline
\end{tabular}

The impact of the zero gas on the measurement of chemical pollutants in air at the requested levels already gives an added uncertainty between $1 \%$ and $5 \%$ relative and it will be higher when the composition of the zero gas cannot be guaranteed. Considering that the maximum allowed uncertainty in fixed measurements for reactive 
gases is $15 \%$ relative at the LVs, having a reliable zero gas standard is deemed necessary.

\subsection{State-of-the-art in zero gas measurements}

In order to evaluate the state-of-the-art in the measurement of zero gases for the measurement of reactive gases in ambient air, a questionnaire was sent out in February 2013 to air quality labs, to National Metrology Institutes (NMIs), to instrument and gas producers. Tables 5 and 6 indicate the capability of measuring reactive impurities $\left(\mathrm{SO}_{2}\right.$ and $\left.\mathrm{NO}_{\mathrm{x}}\right)$ by the target groups. This capability is expressed as limit of detection $(\mathrm{nmol} / \mathrm{mol})$ for the measurement of a specific compound. From the results it seems that, on average, there is a gap between the measurement capabilities of gas producers and of air quality labs, the latter being able to achieve better detection limits.

Table 5. Capability of measuring traces of $\mathrm{SO}_{2}$ in zero gas. In general the used measurement technique is fluorescence, with exception of $*$ and \# where respectively laser spectroscopy and accumulation + ion chromatography are applied.

\begin{tabular}{|c|c|c|c|c|}
\hline \multirow{2}{*}{$\mathrm{SO}_{2}$} & \multicolumn{4}{|c|}{ Detection Limit (nmol/mol) } \\
\hline Respondent & $\begin{array}{c}\text { Air Quality } \\
\text { labs }\end{array}$ & NMIs & $\begin{array}{c}\text { Instrument } \\
\text { Manuf. }\end{array}$ & $\begin{array}{c}\text { Gas } \\
\text { Manuf. }\end{array}$ \\
\hline $\mathrm{A}$ & 0.05 & 0.05 & $0.025_{\#}$ & 0.5 \\
\hline $\mathrm{B}$ & $<0.5$ & $0.5^{*}$ & & 5 \\
\hline $\mathrm{C}$ & 0.5 & 1 & & 10 \\
\hline $\mathrm{D}$ & $<1$ & 3 & & \\
\hline $\mathrm{E}$ & $<1$ & & & \\
\hline
\end{tabular}

Table 6. Capability of measuring traces of $\mathrm{NO}_{\mathrm{x}}$ in zero gas. In general the used measurement technique is chemiluminiscence, with exception of $*$ where laser spectroscopy is applied.

\begin{tabular}{|c|c|c|c|c|}
\hline $\mathrm{NO}_{\mathrm{x}}$ & \multicolumn{4}{|c|}{ Detection Limit (nmol/mol) } \\
\hline Respondent & $\begin{array}{c}\text { Air Quality } \\
\text { labs }\end{array}$ & NMIs & $\begin{array}{c}\text { Instrument } \\
\text { Manuf. }\end{array}$ & $\begin{array}{c}\text { Gas } \\
\text { Manuf. }\end{array}$ \\
\hline $\mathrm{A}$ & 0,05 & 0,1 & 0,35 & 0,4 \\
\hline $\mathrm{B}$ & $<0,4$ & $0,5^{*}$ & 10 & 5 \\
\hline $\mathrm{C}$ & 0,5 & 0,4 & & 5 \\
\hline $\mathrm{D}$ & 0,5 & 1 & & 10 \\
\hline $\mathrm{E}$ & $<1$ & & & \\
\hline
\end{tabular}

\subsection{Measurement approach}

A number of conventional and new optical techniques, considered to be suitable for trace analysis of the impurities listed in the documentary standards, have been tested. These techniques were used to perform measurement of impurities in zero gas at $\mathrm{ppb}$ and sub-ppb level.

At VSL, traceable measurements of $\mathrm{NO}_{2}$ in nitrogen zero gas were carried out with a chemiluminiscence analyser and with a new developed Broadband Cavity Enhanced Absorption Spectroscopy (BB-CEAS) equipment (figure 1). While chemiluminiscence is the reference method for the analysis of $\mathrm{NO}_{\mathrm{x}}$ in ambient air, the other technique is based on the strong and specific adsorption of $\mathrm{NO}_{2}$ around $450 \mathrm{~nm}$. A relatively inexpensive blue led light was used as source, while an absorption path of $5 \mathrm{~km}$ was obtained by means of an optical cavity formed by two highly reflective mirrors. This research demonstrated that both methods, if properly applied, can be used for quantification of $\mathrm{NO}_{2}$ impurities in zero gas at the specification level of $1 \mathrm{nmol} / \mathrm{mol}$. The limit of detection is in fact equal or below $0.1 \mathrm{nmol} / \mathrm{mol}$ and they show a good linear response in the range 1 to 10 $\mathrm{nmol} / \mathrm{mol}$. A similar research was performed for $\mathrm{NO}$ and $\mathrm{SO}_{2}$ impurities.

In addition, measurement strategies, based on spectroscopic techniques, were investigated to detect different impurity species simultaneously.

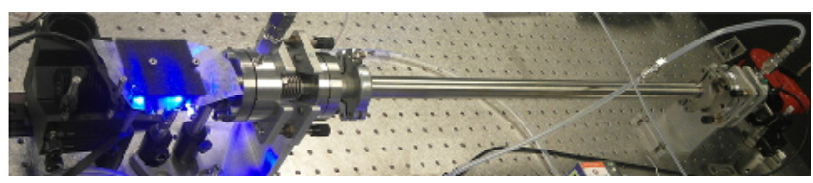

Figure 1. VSL Broadband CEAS for the measurement of $\mathrm{NO}_{2}$ at trace levels. Source: S. Persijn, VSL.

\subsection{Developing a certification protocol}

In order to develop a reliable zero gas standard, a protocol for certification has to be established. This protocol, currently in preparation, makes use of the accurate measurement approaches validated in this project for reactive impurities. In addition, commercial purification systems, filters or scrubbers normally used to remove impurities from nitrogen and air generators, are tested and their removal efficiency evaluated.

\subsection{Case study: adsorption of trace levels of ammonia on material surfaces}

Ammonia is a reactive compound and a potential zero gas impurity. In general ammonia is difficult to measure accurately due to its adsorption on the material surfaces of the measuring systems. This JRP aimed at investigating this effect when ammonia is present at trace levels by means of an almost real-time cavity-ring-down spectroscopy (CRDS). 
The work carried out by the researcher of Helsinki University consisted of building a measuring set-up for ammonia by using a sensitive cavity ringdown spectrometer in the $1.5 \mathrm{~mm}$ spectral region (near infrared) with very high temporal resolution $(<1 \mathrm{~s})$. The experiments performed on different material surfaces (stainless steel, silicon-coated and polymers) confirm other studies and show that ammonia adsorbs greatly to metal surfaces and significantly less in coated or polymer surfaces [8]. This effect is shown in figure 2, where ammonia $(\leq 1 \mu \mathrm{mol} / \mathrm{mol})$ is let through respectively a metal and coated metal tubing of same geometry and its concentration is followed in the time.

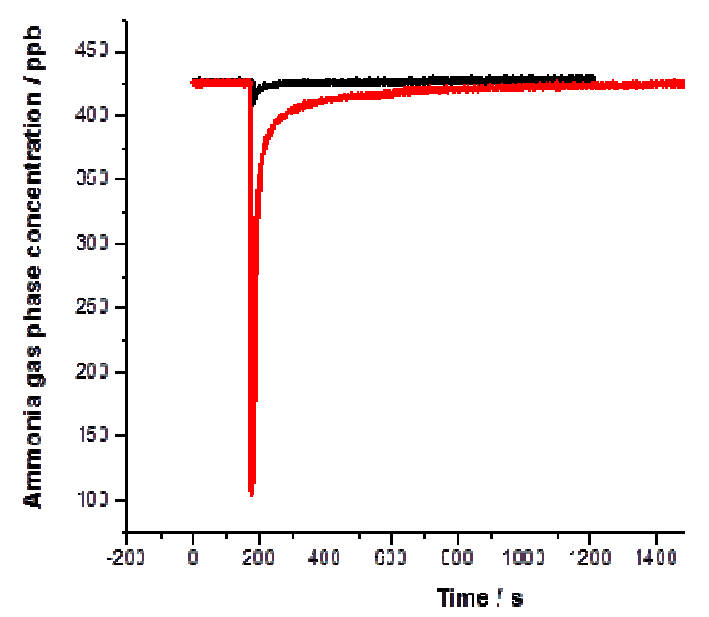

Figure 2. Measurement of adsorption of trace levels of ammonia $(\leq 1 \mu \mathrm{mol} / \mathrm{mol})$ on metal (red) and coated (black) surfaces. Source: O. Vaittinen, University of Helsinki.

\section{Transfer standards for VOC and SVOC in indoor air measurements}

The aim of this part of the project is to prepare $(\mathrm{S}) \mathrm{VOC}$ standards for indoor air applications in the form of transfer sorbent tubes and of constant emitting materials.

The list of compounds under investigation is reported in table 7 . The criterion for selection was their relevance in indoor air monitoring.
Table 7. List of selected (S)VOCs, CAS Numbers and boiling points

\begin{tabular}{|c|c|c|}
\hline Compound & CAS No. & bp, ${ }^{\circ} \mathbf{C}$ \\
\hline Hexadecane & $544-76-3$ & 287 \\
\hline $\begin{array}{c}\text { Dibutylphthalate } \\
\text { (DBP) }\end{array}$ & $84-74-2$ & 340 \\
\hline $\begin{array}{c}\text { Dimethylphthalate } \\
\text { (DBP) }\end{array}$ & $131-11-3$ & 283 \\
\hline 2-Ethyl-1-hexanol & $104-76-7$ & 185 \\
\hline $\begin{array}{c}\text { 1-Methyl-2- } \\
\text { pirrolidone }\end{array}$ & $872-50-4$ & 202 \\
\hline Styrene & $100-42-5$ & 145 \\
\hline
\end{tabular}

Volatile organic compounds (VOCs) and in particular the semi volatiles (SVOCs), i.e. compounds with boiling points between $240-260{ }^{\circ} \mathrm{C}$ to $380-400{ }^{\circ} \mathrm{C}$ according to ISO 16000-6 [9], are of interest also because of the lack of standards for traceability measurements. Some of these compounds are harmful and they are regulated in Europe and/or at national level. Others were suggested by the German Committee for Health Evaluation of Building Products $(\mathrm{AgBB})$ that is responsible of testing the emissions of building products entering the German market.

Hexadecane marks the limit between VOC and SVOC. It is present in many building products like paints, flooring material, etc. This compound is often measurable in a cluster of alcanes. Dibutyl phthalate (DBP) is a plasticizer. It is identified in ECHA list [10] as compound of very high concern and it is strictly limited by French law [11]. Dimethyl phthalate (DMP) is another plasticizer. 2-ethyl-1-hexanol is an alcohol that can be found in carpets and electronic devices. 1-methyl-2pyrrolidone is emitting from flooring material PVC-type. It is identified as compound of very high concern (ECHA list). Styrene is a VOC used as reference compound for checking the accuracy of the measurements carried out in this research.

\subsection{Preparing and comparing transfer standards for (S)VOC}

Suitable techniques for the generation of gas standard of these selected compounds were identified and preliminarily tested. The generation of gas mixtures of these compounds and in particular of dibutyl phthalate is challenging due to their relative low vapour pressures.

The generation of (S)VOC gas mixtures (standard atmospheres) is generally done by dynamic dilution methods. For this research, the selected method was the continuous injection realised according to ISO 6145-part 4 [5]. In addition, the gravimetric method, according to ISO 6142 [12], was applied for the preparation of 
standards containing selected compounds: styrene, 2ethyl-1hexanol and 1-methyl-2-pirrolidone.

The preparation of transfer standards is obtained by loading sorbent tubes with accurately known volumes of (S)VOC gas mixtures. The selected adsorption material for the sorption tubes is tenax $T A \AA$ (60-80 mesh). Although the target is to prepare standards comparable to ambient concentrations in the range of $1-10 \mu \mathrm{g} / \mathrm{m}^{3}$, the work carried out so far aimed at concentrations of one order of magnitude higher.

The feasibility study carried out at VSL showed that all compounds, with exception of DBP, could be evaporated as a mixture, no reactions effects between the compounds have been observed. Approximately $35 \%$ of the expected concentration of DBP generated remains in vapor phase.

The validation study included the verification of the loaded standard tubes against samples spiked by liquid injection with known (S)VOC solutions. There is a good agreement, within $2-5 \%$ for styrene and $n$-hexadecane. For 2-Ethyl-1-hexanol and DMP the agreement is within $7-15 \%$, while for 1-methyl-2-pirrolidone it is within 20$25 \%$. The large variation of the latter compound is mainly due to analytical issues, being the peak shape of the compound difficult to integrate by $\mathrm{GC}$ analysis.

Next step of research in this field is to lower the target (S)VOC concentrations to $10 \mu \mathrm{g} / \mathrm{m}^{3}$.

\subsection{Developing constant emitting reference materials}

The research activities related to indoor air monitoring included the development of a suitable material that enables the emission of (S)VOC compounds at a constant rate. This material is intended to be used for the quality control and quality assurance in emission testing.

The experimental design and research work is performed at BAM.

In order to be able to prepare a constant emitting material, a lacquer type material that acts as support interface for the $(\mathrm{S}) \mathrm{VOC}$ has been proposed. The advantage of this material is the high homogeneity of the organic compound mixture, the relatively fast creation of a dry surface and the slow release of compound emissions from the lacquer film. For the selection of the best suitable support lacquer, twelve type of lacquers, based on acryl (water as solvent) and on alkyd (organic solvents) polymers were tested. The acryl lacquer, transparent and with glossy surface was identified as the best suited carrier material. In particular, it formed a good homogeneous surface during the curing process.

These preliminary tests show that emissions of VOC compounds spiked into this lacquer can be reproduced, at least in a small scale environmental chamber ( $\mu$-CTE), with a variation of less than $10 \%$.

\section{Sensors for air monitoring}

The last part of research performed in MACPoll is related to the development and validation of micro-sensors for air monitoring purposes.

The objectives consist of producing new sensitive micro-sensors (graphene based) for $\mathrm{NO}_{2}$ monitoring and of running an extended programme validation of sensors for $\mathrm{NO}_{2}$ and ozone monitoring. These gases keep on being registered as airpollutants exceeding the European Directive limit values.

\subsection{Developing graphene sensors for $\mathrm{NO}_{2}$}

Currently, two types of graphene sensors, based on exfoliated and epitaxial graphene, are designed and under development by respectively INRIM and MIKES (in collaboration with Aalto University) for the measurement of ambient levels of $\mathrm{NO}_{2}$ (figure 3 ). The performance of these innovative prototype sensors is evaluated and compared to that of the existing sensors.

Moreover, strategies for adding specificity to the sensor (either by direct chemical functionalisation of graphene or by deposition of thin films on graphene) are under investigation.

Details of this work can be found in the web conference publication 'Characterisation of epitaxial and CVD graphene with double metal-graphene contacts for gas sensing' under the $16^{\text {th }}$ International Congress of Metrology [13].

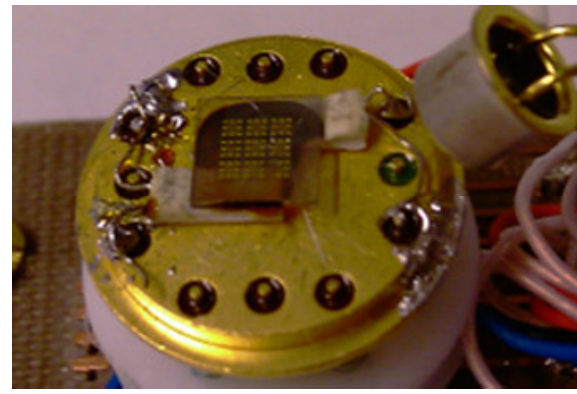

Figure 3: Sensor design based on epitaxial $\mathrm{SiC}$ graphene film. Source: A. Satrapinski, MIKES and S. Novikov, Aalto University.

\subsection{Validating small scale sensors}

Two kinds of commercial sensors have been selected for validation: resistive sensors with high sensitivity but problems of stability and selectivity and electrochemical sensors that present a lower sensitivity but better stability and selectivity to the different gases.

The work activities firstly focused on defining calibration procedures and on developing a testing protocol that takes into account the phenomena involved in the sensor detection process. This research is primarily carried out by JRC in collaboration with a CSIC researcher.

The testing protocol is currently applied in laboratory and in field conditions by exposing micro-sensors to 
different climate conditions, interferents, etc. Beside the evaluation of the sensor response to calibration, other tests carried out are time response, hysteresis, drift and warm up, repeatability, meteorological dependence (temperature and humidity) and interference gases. Figure 4 shows the effect of temperature on the response of a sensor for ozone. As it can be seen, while the ozone concentration measured by the reference system (UV photometer) remains constant at temperature variations, the ozone sensor shows changes in resistivity and a decrease in read-out at the increase of temperature.

Once the experimental part is concluded, parametric and physical models will be verified in order to establish the suitability and uncertainty of the sensor for the specific gas pollutant.

A laboratory validation of 11 selected micro sensors for the measurement of ozone has been performed based on a self-designed validation and calibration protocols. At this stage, the same validation procedure is being applied to $\mathrm{NO}_{2}$ sensors.

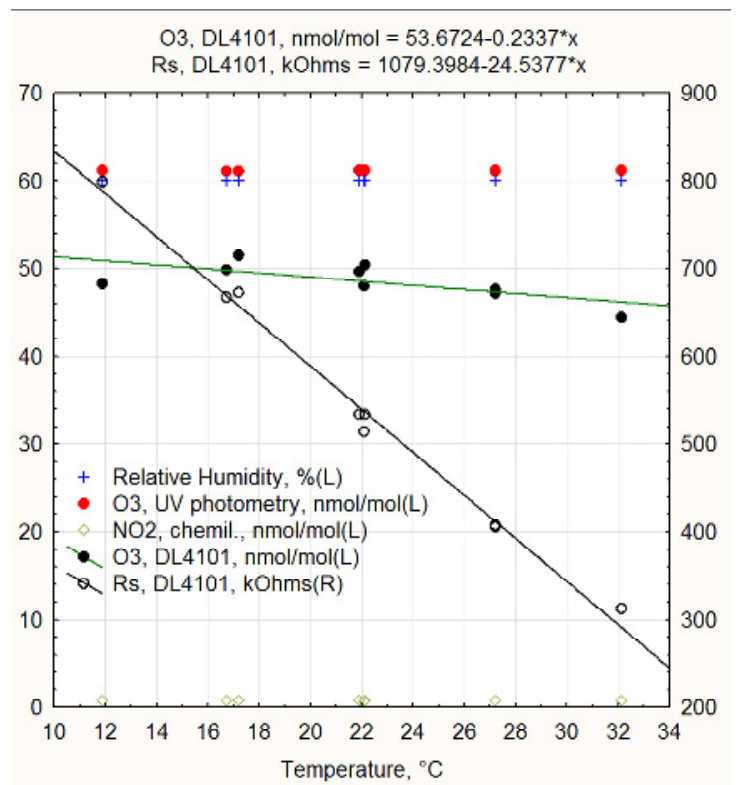

Figure 4: Effect of temperature variation on the read-out of a micro-sensor for ozone. Source: M. Gerboles, L. Spinelli, JRC, EC.

\section{Acknowledgments}

This project, contract number ENV01 MACPoll, runs under the European Metrology Research Programme, a Programme of EURAMET. The EMRP is jointly funded by the EMRP participating countries within EURAMET and by the European Union.

The following scientists must be acknowledged for their inputs in this JRP:

S. Persijn, D. Heikens, J. Li and G. Nieuwenkamp (VSL, the Netherlands), T. Macé, C. Sutour and C. Kaiser (LNE, France), M. Richter, W. Horn and M. Nohr (BAM, Germany), M. Gerboles and L. Spinelli (JRC, Eurpean Commission), J. Walden (FMI, Finland), M. Sega (INRIM, Italy), B. Niederhauser (METAS, Switzerland), M. Vainio and A. Satrapinski (MIKES, Finland), P. Brewer, M. Corbel and M. Doval Minarro
(NPL, United Kingdom), O. Werhahn and A. Pogány (PTB, Germany), V. Štovčík (SMU, Slovak Republic), K. Wirtz and H. Gerwig (UBA, Germany), M. Alexaindre (CSIC, Spain), O. Vaittinen (Helsinki Un., Finland), S. Novikov (Aalto Un., Finland), T. tarhan (TUBITAK, Turkey), N. Skundric (MDMD, Serbia).

\section{References}

1. European Directive 2008/50/EC on 'ambient air quality and cleaner air for Europe', (2008)

2. ENV01 JRP MACPoll, Metrology for Chemical Pollutants in Air, EMRP Programme, (2010-2014)

3. T. Macé et al., 'Calibration gases for existing air quality directive pollutants at Limit Values (LVs)', 16th International Congress of Metrology, EDP Sciences Web of Conferences, (2013)

4. International Organization for Standardization, "ISO 6144, 'Gas analysis - Preparation of calibration gas mixtures - Static volumetric method', ISO Geneva, (2006)

5. International Organization for Standardization, "ISO 6145, 'Gas analysis - Preparation of calibration gas mixtures using dynamic volumetric methods- parts 1-11', ISO Geneva

6. European Committee for Standardization, 'EN 14211 Ambient air - Standard method for the measurement of the concentration of nitrogen dioxide and nitrogen monoxide by chemiluminescence', (2012)

7. European Committee for Standardization, EN 14212Ambient air - Standard method for the measurement of the concentration of sulphur dioxide by ultraviolet fluorescence', (2012)

8. O.Vaittinen, M. Metsälä, S. Persijn, M. Vainio, L. Halonen, 'Adsorption of ammonia on treated stainless steel and polymer surfaces', Appl. Physics B: Lasers and Optics (accepted for publication)

9. International Organization for Standardization, "ISO 16000-6, 'Indoor air -Part 6: Determination of volatile organic compounds in indoor and test chamber air by active sampling on Tenax TA sorbent, thermal desorption and gas chromatography using MS or MS-FID', ISO Geneva (2011)

10. ECHA list, 'Candidate List of Substances of Very High Concern for Authorisation', European Chemical Agency, http://echa.europa.eu/nl/candidate-list-table (last update 20 June 2013)

11. Grenelle Environnement, http://www.developpementdurable.gouv.fr/-Le-Grenelle-de-l-environnement-de.html, France (2007)

12. International Organization for Standardization, "ISO 6142, 'Gas analysis - Preparation of calibration gas mixtures - Gravimetric method', ISO Geneva (2001) 13. S. Novikov et al., 'Characterisation of epitaxial and CVD graphene with double metal-graphene contacts for gas sensing', 16th International Congress of Metrology, EDP Sciences Web of Conferences, (2013) 